Editor's Note: These short, critical reviews of recent papers in the Journal, written exclusively by graduate students or postdoctoral fellows, are intended to summarize the important findings of the paper and provide additional insight and commentary. For more information on the format and purpose of the Journal Club, please see http://www.jneurosci.org/misc/ifa_features.shtml.

\title{
Kalirin-7: Linking Spine Plasticity and Behavior
}

\author{
Julia E. Sommer ${ }^{1,3 *}$ and Elaine C. Budreck ${ }^{2,3 *}$ \\ Departments of ${ }^{1}$ Physiology and Cellular Biophysics and ${ }^{2}$ Neuroscience, Columbia University, College of Physicians and Surgeons, New York, New York \\ 10032, and ${ }^{3}$ Department of Cell Biology, Biozentrum, University of Basel, 4056 Basel, Switzerland \\ Review of Ma et al. (http://www.jneurosci.org/cgi/content/full/28/47/12368)
}

Dendritic spines form the postsynaptic compartment of the majority of excitatory glutamatergic synapses in the brain. Morphological properties of dendritic spines are linked with functional properties of the synapse: e.g., changes in synaptic strength are often accompanied by changes in spine size. This structural and functional plasticity is thought, in part, to underlie the processes of learning and memory. Consistent with a critical role for dendritic spines in human cognition, aberrancies in spine morphology are associated with several neurodevelopmental and neuropsychiatric disorders. Therefore, understanding the molecular factors that contribute to the regulation of dendritic spines is of great interest.

Mechanistically, spine formation and plasticity are driven primarily by rearrangements of the actin cytoskeleton. Actin dynamics are regulated by small GTPases of the Rho family: RhoA, Rac1, and Cdc42. These in turn are modulated by GTP exchange factors (GEFs) and GTPase activating proteins (GAPs) that activate and inactivate Rho GTPases, respec-

Received Jan. 15, 2009; accepted Feb. 12, 2009.

J.E.S. is supported by a PhD fellowship from the Boehringer Ingelheim Fonds, Germany, and E.C.B. is supported by a Ruth L. Kirschstein National Research Service Award from the National Institute of Mental Health (F30MH083473). We thank our mentor Dr. Peter Scheiffele for enthusiastic discussion and helpful comments on this manuscript.

*J.E.S. and E.C.B. contributed equally to this work.

Correspondence should be addressed to either Julia E. Sommer or Elaine C. Budreck, Biozentrum, University of Basel, Klingelbergstrasse 70, 4056 Basel, Switzerland, E-mail: Julia.Sommer@unibas.ch or Elaine.Budreck@ unibas.ch.

D0I:10.1523/JNEUROSCI.0235-09.2009

Copyright $\odot$ 2009 Society for Neuroscience $\quad$ 0270-6474/09/295367-03\$15.00/0 tively. Rho-GEFs and Rho-GAPs are downstream effectors of a variety of synaptic signaling pathways. They are thereby poised to link transsynaptic signaling events with the actin cytoskeleton to coordinate changes in dendritic spine morphology with alterations in neuronal activity. Over 60 GEFs have been identified in the mouse genome, and recent studies have suggested important roles for several in spine morphogenesis in vitro. Because of possible overlapping functions of these Rho regulators, a recent challenge has been to determine the specific roles of such factors in the intact nervous system.

One promising candidate to serve a critical function in vivo is kalirin-7, a Rac1-GEF expressed postnatally in neurons and localized to the postsynaptic density. In vitro studies using cultured neurons have demonstrated that overexpression of kalirin-7 induces the formation of dendritic spines, whereas knockdown or loss of function of kalirin-7 leads to a decrease in spines (Penzes et al., 2001; Ma et al., 2003). Kalirin-7 acts downstream of synaptic adhesion molecules, for example the ephrinB-EphB complex and $\mathrm{N}$-Cadherin, and is involved in activity-dependent spine plasticity and recruitment of AMPA receptors (AMPARs) into spines (Penzes et al., 2003; Xie et al., 2007, 2008). Whether kalirin-7 is required in vivo for spine formation and dynamics that ultimately may underlie learning and memory had not been previously studied. To address these questions, Ma et al. (2008) generated kalirin-7 knock-out (kal7 KO) mice. In a recent issue of The
Journal of Neuroscience, they described the analysis of spine and synapse morphology, functional electrophysiology, and behavior of these mice.

The authors first examined whether kalirin-7 is required for the formation/ maintenance of dendritic spines and synapses in vivo by analyzing CA1 hippocampal pyramidal neurons from adult kal7 $\mathrm{KO}$ and wild-type (WT) mice. Consistent with previous in vitro studies, by Golgi staining, they detected a decrease in spine density in kal7 KOs to $85 \%$ of WT levels [Ma et al. (2008), Fig. 2]. Furthermore, analysis of electron micrographs revealed a $30 \%$ reduction in the density of glutamatergic synapses [Ma et al. (2008), their Fig. 7], confirming the loss of spines is accompanied by decreases in synaptic structures. Corroborating these in vivo observations, the authors found that the density of glutamatergic synapses in cortical neurons cultured from kal7 KOs was reduced by $39 \%$ compared with WT neurons [Ma et al. (2008), their Fig. 9].

Next, to investigate whether the morphological alterations in the kal7 KOs lead to defects in synaptic function, $\mathrm{Ma}$ et al. (2008) performed electrophysiological experiments using acute hippocampal slices. They found a decrease in spontaneous EPSC frequency but not amplitude in the kal-7 KOs, as well as an unaltered evoked EPSC amplitude [Ma et al. (2008), their Fig. 4]. Together, these data indicate that despite the reduction in synapse number, baseline synaptic transmission remains intact in the absence of kalirin-7. Furthermore, to test for a role of kalirin-7 


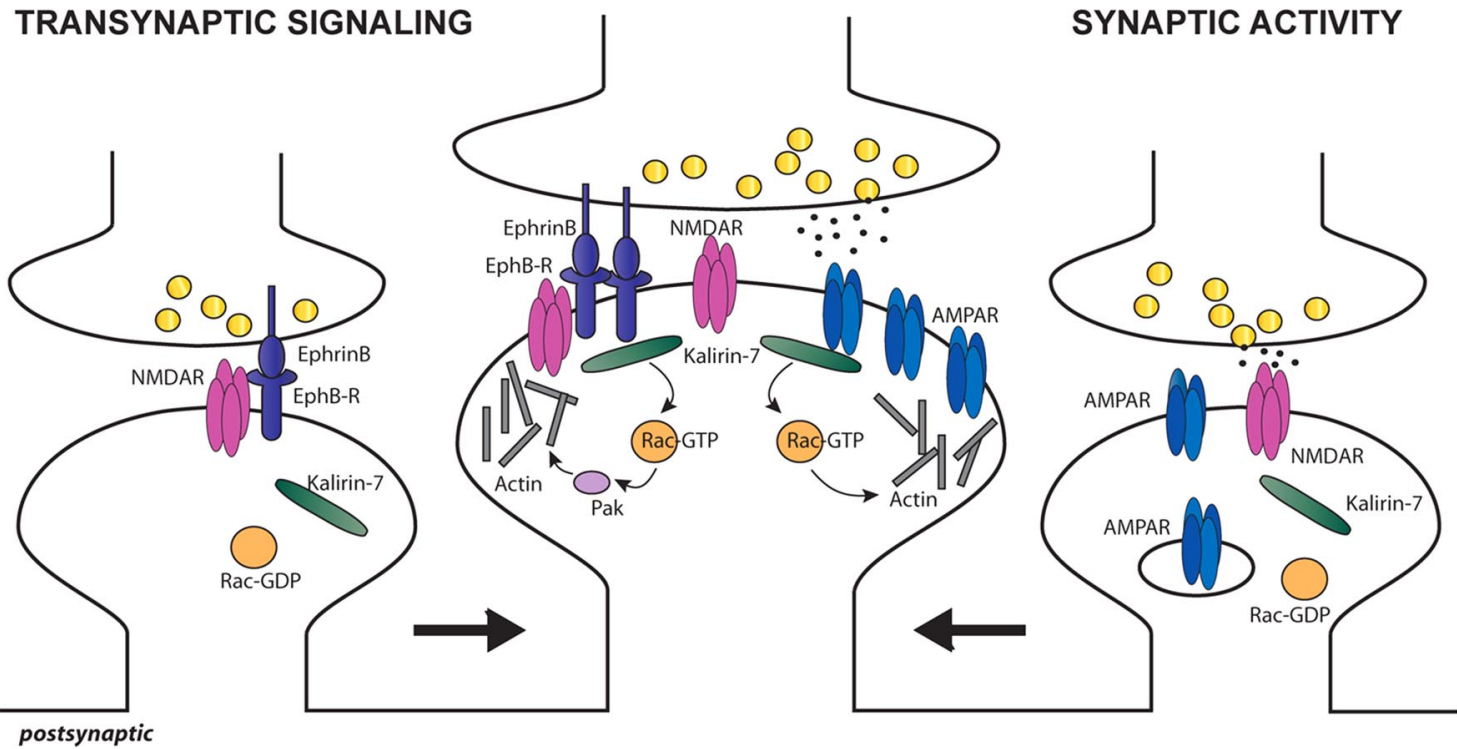

Figure 1. Kalirin-7-mediated alterations in dendritic spine morphology. Kalirin-7 can lead to increases in spine number (not shown) and size downstream of transsynaptic signaling induced by ephrinB stimulation of EphB receptors (on left) and/or enhanced neuronal activity (on right). During activation of EphB, kalirin-7 is recruited and activates Rac1, leading to Pak-dependent actin polymerization. Neurotransmitter release activating NMDA receptors results in kalirin-7-mediated Rac1-dependent actin polymerization and synaptic AMPAR insertion. In both scenarios, actin polymerization leads to spine enlargement.

in synaptic plasticity, a theta burst pairing paradigm to elicit long-term potentiation (LTP) was used. Although the induction of LTP was not affected in kal7 KOs, the magnitude of LTP was significantly smaller compared with WT. Expression of LTP depends on synaptic insertion of AMPARs; therefore, the decreased LTP in the mutants might reflect a deficit in AMPAR trafficking. Previous in vitro studies have implicated kalirin-7 in activitydependent synaptic AMPAR localization (Xie et al., 2007). The current results complement the in vitro findings and support a requirement for kalirin-7 in synaptic plasticity in vivo.

Although Kal7 KO mice were viable, fertile, and had intact gait, reflexes, and motor function, they exhibited diminished anxiety compared with WT mice. Consequently, the authors questioned whether the observed synaptic disruptions might additionally lead to more specific deficits in hippocampal-dependent behaviors and therefore subjected adult kal7 KO and WT mice to a number of assays probing learning and memory. Their most intriguing finding was a selective disruption in specific aspects of learning in the kal7 KOs [Ma et al. (2008), their Fig. 3]. Kal7 KO mice performed no differently from WT in a reward-dependent memory task (radial arm maze) or in an object recognition test. In contrast, they exhibited an impairment in contextual fear memory, as assessed by a paradigm in which learning is associated with an aver- sive stimulus. Thus, lack of kalirin-7 results in specific impairments in anxiety and fear-based learning, rather than in general perturbations of learning ability.

Together, in this series of experiments, Ma et al. (2008) have importantly established for the first time the requirement for kalirin-7 not only for spine formation/ maintenance, but also for proper synaptic function and behavior in vivo. This study nicely shows that KO of kalirin-7 recapitulates the spine phenotype observed in in vitro studies, demonstrating that despite the existence of many Rho-GEFs in brain, kalirin-7 performs an essential function in vivo. An open question for future study will be to determine the mechanisms by which loss of kalirin-7 in vivo leads to morphological and functional alterations of spines and synapses.

In vitro evidence strongly links kalirin-7 to pathways regulating actin cytoskeletal dynamics that govern dendritic spine growth. Kalirin-7 activation of the small GTPase Racl can lead to the activation of p21 activated kinase (Pak) (Penzes et al., 2003) and subsequent Pakdependent induction of actin polymerization. Accordingly, the reduction in spine density observed upon loss of kalirin-7 could arise from decreases in active Racl and Pak. Although global levels of activated Racl were unaltered in kal7 KOs [Ma et al. (2008), their Fig. 5], it is likely that kalirin-7 controls localized transient activation of Racl that requires more sensitive methods to be detected. Interest- ingly, the $\mathrm{KO}$ of kalirin-7 leads to a decrease in the levels of synaptic cyclindependent kinase 5 (Cdk5) [Ma et al. (2008), their Fig. 8], which has also been implicated in the regulation of the actin cytoskeleton downstream of Racl. In vitro studies have demonstrated that Cdk5 phosphorylates and thereby inactivates Pak in a Rac-GTP-dependent manner (Nikolic et al., 1998). The opposing actions of kalirin-7 and Cdk5 on the activity of Pak could allow for rapid rearrangements in the actin cytoskeleton. However, why the loss of kalirin-7 results in a reduction of synaptic Cdk5 levels is currently unclear. As suggested by the authors, the decrease in synaptic Cdk5 might contribute to the phenotype of the kal7 KOs. In future studies, it will, therefore, be important to reconstitute Cdk5 to WT levels in kal7 $\mathrm{KO}$ neurons to test whether the $\mathrm{KO}$ phenotype is reversed.

The signals upstream of kalirin-7 that lead to the activation of the Rac/Pak/actin pathway in vivo are currently unknown. The ephrinB-EphB2 transsynaptic protein complex represents a potential candidate for this function (Fig. 1). In vitro dendritic spine growth triggered by activation of EphB2 is dependent on the recruitment of kalirin-7 and activation of Pak (Penzes et al., 2003). Additionally, the decreased spine density observed in EphB1/2/3 triple $\mathrm{KO}$ mice is reminiscent of the kal7 $\mathrm{KO}$ phenotype and therefore is consistent with the involvement of EphB2 upstream of kalirin-7 in this pathway in vivo (Hen- 
kemeyer et al., 2003 and Kayser et al., 2006). To determine whether ephrinBEphB signaling indeed acts upstream of kalirin-7, one could test if ephrinBinduced spine alterations are perturbed in kal7 KO neurons.

Alternatively, the loss of spines and synapses in the kal7 KOs could be a consequence of the defects in synaptic plasticity. Strengthening of synapses as induced by LTP paradigms involves a coordinate increase in dendritic spine size and synaptic insertion of AMPARs. Failure of synapses to respond to activity in this way can result in their destabilization and elimination. Kalirin-7 has been implicated in Rac1-dependent spine enlargement and synaptic AMPAR insertion in response to enhanced neuronal activity (Xie et al., 2007) (Fig. 1). Therefore, it would be interesting to examine activity-induced trafficking of AMPARs in kal7 KOs to determine if a disruption in this process might underlie the LTP impairment and spine loss observed in the KO.

A remaining question raised by this study is how the loss of kalirin-7 can result in specific deficits in anxiety and contextual fear memory while leaving other behaviors intact. One possible explanation could be selective regional expression of kalirin-7, leading to the activation of specific circuits underlying fear-based learn- ing. Kalirin-7 is enriched in brain regions typically associated with learning: the hippocampus and the cortex. However, such broad expression cannot easily account for a discrete role of kalirin-7 in fearbased learning, in particular. An alternate possibility would entail specific induction of kalirin-7-dependent signal transduction pathways in response to aversive stimuli. In this scenario, kalirin-7 would be essential to induce synaptic modifications downstream of signals evoked by aversive learning paradigms. According to this hypothesis, other Rho-GEFs could similarly engage in selective signal transduction pathways underlying specific behaviors. In the future, generation and analysis of additional Rho-GEF KOs will inform if this concept is indeed more broadly applicable.

Although these questions will be interesting to address in the future, this study by Ma et al. (2008) defines a critical role of the Rho-GEF kalirin-7 in vivo and importantly contributes to a better understanding of the link between spine morphology, synaptic plasticity, and behavior.

\section{References}

Henkemeyer M, Itkis OS, Ngo M, Hickmott PW, Ethell IM (2003) Multiple EphB receptor tyrosine kinases shape dendritic spines in the hippocampus. J Cell Biol 163:1313-1326.

Kayser MS, McClelland AC, Hughes EG, Dalva MB (2006) Intracellular and trans-synaptic regulation of glutamatergic synaptogenesis by EphB receptors. J Neurosci 26:12152-12164.

Ma XM, Huang J, Wang Y, Eipper BA, Mains RE (2003) Kalirin, a multifunctional Rho guanine nucleotide exchange factor, is necessary for maintenance of hippocampal pyramidal neuron dendrites and dendritic spines. J Neurosci 23:10593-10603.

Ma XM, Kiraly DD, Gaier ED, Wang Y, Kim EJ, Levine ES, Eipper BA, Mains RE (2008) Kalirin-7 is required for synaptic structure and function. J Neurosci 28:12368-12382.

Nikolic M, Chou MM, Lu W, Mayer BJ, Tsai LH (1998) The p35/Cdk5 kinase is a neuronspecific Rac effector that inhibits Pak1 activity. Nature 395:194-198.

Penzes P, Johnson RC, Sattler R, Zhang X, Huganir RL, Kambampati V, Mains RE, Eipper BA (2001) The neuronal Rho-GEF kalirin-7 interacts with PDZ domain-containing proteins and regulates dendritic morphogenesis. Neuron 29:229-242.

Penzes P, Beeser A, Chernoff J, Schiller MR, Eipper BA, Mains RE, Huganir RL (2003) Rapid induction of dendritic spine morphogenesis by trans-synaptic ephrinB-EphB rexeptor ativation of the Rho-GEF kalirin. Neuron 37:263-274.

Xie Z, Srivastava DP, Photowala H, Kai L, Cahill ME, Woolfrey KM, Shum CY, Surmeier DJ, Penzes P (2007) Kalirin-7 controls activitydependent structural and functional plasticity of dendritic spines. Neuron 56:640-656.

Xie Z, Photowala H, Cahill ME, Srivastava DP, Woolfrey KM, Shum CY, Huganir RL, Penzes P (2008) Coordination of synaptic adhesion with dendritic spine remodeling by AF- 6 and kalirin-7. J Neurosci 28:6079-6091. 